\title{
Incidência e manejo da neutropenia em pacientes submetidas ao protocolo AC-T no
}

\section{tratamento adjuvante de câncer de mama}

\author{
Incidence and management of neutropenia in patients undergoing the $\mathrm{AC}$ - $\mathrm{T}$ protocol in the \\ adjuvant treatment of breast cancer
}

Incidencia y manejo de la neutropenia en pacientes sometidas al protocolo AC-T en el tratamiento adyuvante del cáncer de mama

Recebido: 12/05/2021 | Revisado: 18/05/2021 | Aceito: 21/05/2021 | Publicado: 07/06/2021

\author{
Luana do Amaral Brasileiro \\ ORCID: https://orcid.org/0000-0001-7683-5512 \\ Instituto Nacional de Câncer, Brasil \\ E-mail: luana.brasileiro@inca.gov.br \\ Júlia Mouta de Oliveira \\ ORCID: https://orcid.org/0000-0002-7808-1467 \\ Instituto Nacional de Câncer, Brasil \\ E-mail: juliamout@gmail.com \\ Selma Rodrigues de Castilho \\ ORCID: https://orcid.org/0000-0003-0272-4777 \\ Universidade Federal Fluminense, Brasil \\ E-mail: selmarc@id.uff.br
}

\begin{abstract}
Resumo
O câncer de mama é o tumor mais incidente entre mulheres. A introdução da quimioterapia antineoplásica sistêmica adjuvante trouxe como benefícios a redução do risco de recorrência e mortalidade por câncer de mama. Porém, intrínseca a toda quimioterapia observa-se a ocorrência de reações adversas, entre elas a neutropenia induzida por quimioterapia, uma reação incidente claramente relacionada a eventos de neutropenia febril. Tais eventos podem gerar impacto significativo nos custos hospitalares e comprometer o objetivo do tratamento quimioterápico adjuvante. Em estudo transversal, descritivo, quantitativo e retrospectivo, 289 pacientes do sexo feminino com câncer de mama e em tratamento quimioterápico sistêmico adjuvante com o protocolo AC-T, foram avaliadas de modo a descrever a incidência de neutropenia e identificar os manejos realizados para manutenção da utilização do tratamento. A incidência bruta de neutropenia foi de 52,2\% durante a aplicação de AC (doxorrubicina e ciclofosfamida), de 26,9\% durante a aplicação de $\mathrm{T}$ (docetaxel) nos pacientes que não fizeram profilaxia primária (PP), e de 15,1\% durante a aplicação de T nos pacientes que fizeram PP. Entre os manejos identificados, uso de antibioticoterapia $(25,4 \%)$, uso de fatores estimuladores de colônia de granulócitos $(23 \%)$ e adiamento de ciclo (20,3\%) foram os mais observados. A ocorrência de eventos de neutropenia ao longo do tratamento quimioterápico para câncer de mama é iminente. Todavia, pode levar a alterações no regime que podem comprometer o tratamento. Neste estudo, a ocorrência de neutropenia, aponta para a necessidade de definir protocolos de utilização de PP e manejo da reação adversa.

Palavras-chave: Neoplasias da mama; Neutropenia; Neutropenia febril induzida por quimioterapia; Efeitos colaterais e reações adversas relacionados a medicamentos.
\end{abstract}

\begin{abstract}
Breast cancer is the most common tumor among women. The introduction of adjuvant systemic antineoplastic chemotherapy has the benefits of reducing the risk of recurrence and mortality from breast cancer. However, intrinsic to all chemotherapy, adverse reactions are observed, including chemotherapy-induced neutropenia, an incident reaction clearly related to febrile neutropenia events. Such events can have a significant impact on hospital costs and compromise the objective of adjuvant chemotherapy treatment. In a cross-sectional, descriptive, quantitative and retrospective study, 289 female patients with breast cancer and undergoing systemic chemotherapy adjuvant with the AC-T protocol, were evaluated in order to describe the incidence of neutropenia and identify the managements performed to maintain the treatment use. The gross incidence of neutropenia was $52.2 \%$ during the application of CA (doxorubicin and cyclophosphamide), 26.9\% during the application of T (docetaxel) in patients who did not undergo primary prophylaxis (PP), and 15, $1 \%$ during application of $\mathrm{T}$ at the patients who made PP. Among the identified managements, use of antibiotic therapy $(25.4 \%)$, use of granulocyte colony stimulating factors $(23 \%)$ and postponement of the cycle $(20.3 \%)$ were the most observed. The occurrence of neutropenia events during chemotherapy for breast cancer is imminent. However, it can lead to changes in the regimen that can
\end{abstract}


compromise treatment. In this study, the occurrence of neutropenia points to the need to define protocols for the use of PP and management of the adverse reaction.

Keywords: Breast neoplasms; Neutropenia; Chemotherapy-induced febrile neutropenia; Drug-related side effects and adverse reactions.

\section{Resumen}

El cáncer de mama es el tumor más común entre las mujeres. La introducción de la quimioterapia antineoplásica sistémica adyuvante tiene los beneficios de reducir el riesgo de recurrencia y mortalidad por cáncer de mama. Sin embargo, intrínsecas a toda quimioterapia, se observan reacciones adversas, incluida la neutropenia inducida por quimioterapia, una reacción incidente claramente relacionada con eventos de neutropenia febril. Tales eventos pueden tener un impacto significativo en los costos hospitalarios y comprometer el objetivo del tratamiento de quimioterapia adyuvante. En un estudio transversal, descriptivo, cuantitativo y retrospectivo, se evaluaron 289 pacientes femeninas con cáncer de mama y sometidas a quimioterapia sistémica adyuvante con el protocolo AC-T, con el fin de describir la incidencia de neutropenia e identificar los manejos realizados para mantener el tratamiento. usar. La incidencia bruta de neutropenia fue del 52,2\% durante la aplicación de AC (doxorrubicina y ciclofosfamida), del 26,9\% durante la aplicación de $\mathrm{T}$ (docetaxel) en pacientes que no se sometieron a profilaxis primaria (PP) y del 15,1\% durante la aplicación de $\mathrm{T}$ a los pacientes que hicieron PP. Entre los manejos identificados, el uso de terapia antibiótica $(25,4 \%)$, el uso de factores estimulantes de colonias de granulocitos (23\%) y el aplazamiento del ciclo (20,3\%) fueron los más observados. La aparición de eventos de neutropenia durante la quimioterapia para el cáncer de mama es inminente. Sin embargo, puede provocar cambios en el régimen que pueden comprometer el tratamiento. En este estudio, la aparición de neutropenia apunta a la necesidad de definir protocolos para el uso de PP y manejo de la reacción adversa.

Palabras clave: Neoplasias de la mama; Neutropenia; Neutropenia febril inducida por quimioterapia; Efectos colaterales y reacciones adversas relacionados con medicamentos.

\section{Introdução}

O câncer de mama é o segundo mais incidente no mundo, representando 11,6\% de todos os novos casos de câncer no ano de 2018. Nas mulheres, é o mais frequente e a principal causa de morte por câncer (Bray et al., 2018). No Brasil, estima-se a ocorrência de aproximadamente 66 mil novos casos para cada ano do triênio 2020-2022 (Instituto Nacional de Câncer José Alencar Gomes da Silva [INCA], 2019).

A introdução da quimioterapia antineoplásica sistêmica adjuvante, nas últimas décadas, trouxe como benefícios a redução do risco de recorrência e mortalidade por câncer de mama. Os esquemas terapêuticos mais utilizados para neoplasia de mama com indicação adjuvante são baseados na associação de antraciclinas (doxorrubicina e epirrubicina), agente alquilante (ciclofosfamida), antimetabólitos (metotrexato e fluoruracila) e taxanos (docetaxel e paclitaxel) (Bines, Earl, Buzaid, \& Saad, 2014; Ho \& Mackey, 2014; Blum et al., 2017; Abolaleb et al., 2018).

O protocolo AC-T consiste na administração de doxorrubicina (A) $60 \mathrm{mg} / \mathrm{m}^{2}$ e ciclofosfamida (C) $600 \mathrm{mg} / \mathrm{m}^{2}$, a cada 21 dias, por 4 ciclos, seguidos de docetaxel (T) $100 \mathrm{mg} / \mathrm{m}^{2}$, a cada 21 dias, por 4 ciclos. Observa-se que a adição de taxanos a antraciclinas traz benefícios de forma não relacionada a fatores como idade, comprometimento de linfonodos, tamanho do tumor, status dos receptores hormonais e do fator de crescimento epidérmico humano tipo 2 (HER2), sendo responsável por redução adicional na taxa de eventos de recorrência de 0,87 e 0,99 na taxa de mortalidade (Ho \& Mackey, 2014; Blum et al., 2017; Cardoso et al., 2019).

Entretanto, apesar dos benefícios clínicos, o efeito citotóxico da quimioterapia não ocorre exclusivamente em células cancerígenas, atingindo também tecidos não tumorais de rápida proliferação, sendo associado à ocorrência de reações adversas (Sociedade Brasileira de Farmacêuticos em Oncologia [SOBRAFO], 2011; Pereira, Nogueira, Barbosa, Calil-Elias, \& Castilho, 2015).

A neutropenia induzida por quimioterapia (NIQ) é uma reação incidente que ocorre principalmente pela supressão do sistema hematopoiético e comprometimento do sistema imunológico do paciente, descrita como uma queda na contagem absoluta de neutrófilos (CAN) circulantes no sangue periférico (Furuya, 2019). Existe clara correlação entre a presença de 
neutropenia severa e eventos de neutropenia febril (NF), uma emergência oncológica que está correlacionada a aumento de morbidade, mortalidade, estadia no hospital, utilização de antibióticos e fatores estimuladores de colônia de granulócitos (GCSFs), consequentemente gerando um impacto significativo nos custos hospitalares (Aras, Bayraktar-Ekincioglu, \& Killickap, 2020).

A neutropenia severa e a NF frequentemente resultam em reduções de dose, atrasos nos intervalos de administração dos ciclos de quimioterapia e interrupção do protocolo de tratamento, podendo comprometer o desfecho clínico, principalmente, quando a finalidade do tratamento é adjuvante (Hansson \& Friberg, 2012; Faqeer et al., 2016; Hegg et al., 2016).

A profilaxia com G-CSFs pode facilitar a administração da dose plena de quimioterapia e o intervalo adequado de administração de acordo com o protocolo planejado (Minckwitz et al., 2009). G-CSFs são fatores biológicos de crescimento hematopoiético que estimulam a proliferação e diferenciação de neutrófilos (Wang, Baser, Kutikova, Page, \& Barron, 2015). As diretrizes internacionais aconselham medidas de profilaxia com G-CSFs para protocolos com risco de desenvolvimento de neutropenia febril superior a 20\%. Em protocolos com risco intermediário (10-20\%), grupo em que se enquadra o protocolo em estudo, recomenda-se avaliação de risco previamente a cada ciclo de quimioterapia (Klastersky et al., 2016; National Comprehensive Cancer Network [NCCN], 2020).

Assim, este trabalho visou descrever a incidência de neutropenia em pacientes com câncer de mama em tratamento adjuvante de quimioterapia com o protocolo AC-T, identificando o manejo realizado para manutenção da utilização deste protocolo frente à neutropenia apresentada.

\section{Metodologia}

Trata-se um estudo transversal, descritivo, quantitativo, com coleta de dados retrospectiva (Pereira, Shitsuka, Pereira, \& Shitsuka, 2018), realizado em um hospital federal do Rio de Janeiro especializado em câncer de mama. O estudo foi aprovado pelo Comitê de Ética em Pesquisa da instituição sob o número CAAE 81843718.5.0000.5274.

Pacientes do sexo feminino, com idade superior a 18 anos, diagnosticadas com câncer de mama e em tratamento quimioterápico sistêmico adjuvante com o protocolo AC-T, com aplicação de docetaxel entre o período de 01 de janeiro de 2015 a 31 dezembro de 2017 foram incluídas no estudo. Os critérios de exclusão foram prontuários não localizados, ausência de registro adequado em evolução médica, ausência de dados laboratoriais com contagem absoluta de neutrófilos antes das aplicações dos ciclos de quimioterapia e realização de quimioterapia ou hormonioterapia prévia.

Os dados das pacientes foram obtidos de prontuários físicos e eletrônicos, sendo incluídas as seguintes variáveis: idade, cor da pele, peso, altura, histórico familiar de câncer, hábitos sociais (tabagismo), comorbidades, medicamentos de uso contínuo, número de ciclos de quimioterapia, contagem absoluta de neutrófilos, presença de neutropenia afebril e/ou NF e seus respectivos manejos, e utilização de G-CSFs como profilaxia primária.

Com base no peso e altura da paciente foi calculado o índice de massa corpórea (IMC). Para o cálculo do IMC, foi utilizada a fórmula padrão que consiste em dividir o peso em quilogramas $(\mathrm{kg})$ pelo quadrado da altura em metros $(\mathrm{m})$. Para classificação da paciente em relação ao seu IMC, foi empregada a proposta das Diretrizes Brasileiras de Obesidade, sendo assim considerado baixo peso se IMC $<18,5 \mathrm{~kg} / \mathrm{m}^{2}$; peso normal se IMC $18,5-24,9 \mathrm{~kg} / \mathrm{m}^{2}$; pré-obeso se IMC $25-29,9 \mathrm{~kg} / \mathrm{m}^{2}$; obeso I se IMC 30 - 34,9 kg/m²; obeso II se IMC 35 - 39,9 kg/m²; e obeso III se IMC $\geq 40 \mathrm{~kg} / \mathrm{m}^{2}$ (Associação Brasileira para o Estudo da Obesidade e da Síndrome Metabólica [ABESO], 2016).

A classificação dos medicamentos utilizados pelos pacientes foi realizada com base na Anatomical Therapeutic Chemical (ATC) (WORLD HEALTH ORGANIZATION [WHO], 2020). 
Uma vez havendo a manifestação da neutropenia, a mesma foi classificada em termos de sua gravidade, de acordo com o proposto pela Sociedade Brasileira de Farmacêuticos em Oncologia (SOBRAFO), sendo assim considerada grau 1 se

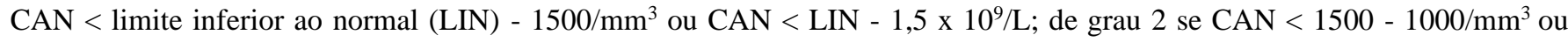
CAN < 1,5 - 1,0 x 109/L; de grau 3 se CAN < $1000-500 / \mathrm{mm}^{3}$ ou CAN < 1,0 - 0,5 x 109 $/ \mathrm{L}$; e de grau 4 se CAN $<500 / \mathrm{mm}^{3}$ ou CAN $<0,5 \times 10^{9} / \mathrm{L}($ SOBRAFO, 2011).

Considerou-se presença de neutropenia febril um episódio de temperatura oral $\geq 38,3^{\circ} \mathrm{C}$ ou $\geq 38,0^{\circ} \mathrm{C}$ durante 1 hora, neutropenia $<500$ neutrófilos $/ \mu \mathrm{L}$ ou $<1.000$ neutrófilos $/ \mu \mathrm{L}$ e um declínio previsto para $\leq 500$ neutrófilos/ $\mu \mathrm{L}$ ao longo das próximas 48 horas (NCCN, 2020).

A população foi tratada inicialmente como um só grupo durante a aplicação da primeira etapa do protocolo, onde há utilização dos medicamentos doxorrubicina e ciclofosfamida (AC). Na segunda parte do protocolo, onde ocorre utilização de docetaxel (T), a população foi dividida em dois grupos, com e sem uso de profilaxia primária (PP). Considerou-se PP o uso de G-CSFs a partir do primeiro ciclo de aplicação de docetaxel e ocorreu de acordo com critérios médicos, uma vez que não havia protocolo institucional para tal conduta. Esses grupos foram analisados separadamente devido ao efeito profilático dos G-CSFs frente a incidência de neutropenia.

Os dados foram compilados em uma base no programa Microsoft Office Excel®. Estes foram processados e analisados pelo programa Statistical Package For Social Sciences (SPSS) - versão 22.0.

As variáveis categóricas tiveram suas frequências calculadas, enquanto as variáveis quantitativas contínuas foram testadas quanto à sua normalidade pelo teste de Kolmogorov-Smirnov e obtidas médias. O Teste-t foi usado para comparação entre variáveis quantitativas, onde valores p bi-caudais inferiores a 0,05 foram considerados estatisticamente significativos.

\section{Resultados e Discussão}

O estudo foi composto por 289 pacientes. As características da população se encontram descritas na Tabela 1.

Tabela 1: Distribuição da população, por idade, cor, hábitos sociais, índice de massa corporal, histórico familiar de câncer, presença de comorbidades e uso de medicamentos $(\mathrm{N}=289)$.

\begin{tabular}{l|c}
\hline Características da população & N (\%) \\
\hline Faixa etária & $7(2,4)$ \\
\hline$\leq$ a 30 anos & $21(7,3)$ \\
\hline 31 a 40 anos & $83(28,7)$ \\
\hline 41 a 50 anos & $106(36,7)$ \\
\hline 51 a 60 anos & $65(22,5)$ \\
\hline 61 a 70 anos & $7(2,4)$ \\
\hline$\geq$ a 71 anos & $119(41,2)$ \\
\hline Cor & $36(12,5)$ \\
\hline Branca & $133(46)$ \\
\hline Parda & \\
\hline Preta & \\
\hline
\end{tabular}


Research, Society and Development, v. 10, n. 6, e48210616018, 2021

(CC BY 4.0) | ISSN 2525-3409 | DOI: http://dx.doi.org/10.33448/rsd-v10i6.16018

\begin{tabular}{l|c}
\hline Não relatada & $1(0,3)$ \\
\hline Hábitos sociais & $212(73,4)$ \\
\hline Não - tabagista & $45(15,6)$ \\
\hline Ex - tabagista & $32(11,1)$ \\
\hline Tabagista & $3(1,0)$ \\
\hline Índice de massa corporal & $56(19,4)$ \\
\hline Baixo peso & $106(36,7)$ \\
\hline Peso normal & $81(28,0)$ \\
\hline Pré-obeso & $34(11,8)$ \\
\hline Obeso I & $9(3,1)$ \\
\hline Obeso II &
\end{tabular}

Histórico familiar de câncer

\begin{tabular}{l|c}
\hline Sim & $184(63,7)$ \\
\hline Não & $69(23,9)$ \\
\hline Não relatado & $36(12,5)$ \\
\hline
\end{tabular}

\section{Presença de comorbidades}

\begin{tabular}{l|c}
\hline Sim & $212(73,4)$ \\
\hline Não & $74(25,6)$ \\
\hline Não relatado & $3(1,0)$ \\
\hline Uso de medicamentos & $170(58,8)$ \\
\hline Sim & $91(31,5)$ \\
\hline Não & $28(9,7)$ \\
\hline Não relatado & \\
\hline
\end{tabular}

Fonte: Autores.

A população analisada possuía idade média de 53 anos, com variação de 25 a 74 anos, sendo 36,7\% enquadradas na faixa etária de 51 e 60 anos, corroborando o descrito por Blum et al. (2017).

Segundo INCA (2019), idade superior a 50 anos é um fator de risco para o desenvolvimento de câncer de mama, juntamente com fatores genéticos, hereditários, menopausa tardia, obesidade, sedentarismo e exposições frequentes a radiações ionizantes.

Constatou-se nesse estudo uma média de idade de 52 anos entre as pacientes que apresentaram neutropenia utilizando o protocolo AC-T, sendo 39,7\% delas com idade entre 51 e 60 anos. Não se encontrou diferença estatisticamente significativa entre a média de idade das pacientes do estudo que não apresentaram neutropenia e aquelas que apresentaram esta reação 
adversa (p=0,08). Embora estudos recentes (Catala et al., 2020; NCCN, 2020) tenham apontado idade $\geq 65$ anos como um fator de risco para o desenvolvimento de neutropenia durante o tratamento quimioterápico, neste estudo não se observou correlação entre este fator e o desenvolvimento desta reação adversa.

Até o presente momento, a cor da pele não é considerada fator de risco para desenvolvimento do câncer de mama.

A análise dos hábitos sociais mostrou que $73,4 \%$ das pacientes se declararam não tabagistas. Apesar de não ser demonstrada correlação entre tabagismo e câncer de mama, tal hábito é relacionado à presença de cânceres de próstata, cólon e reto, pulmão, útero, cavidade oral, esôfago, tireóide, bexiga e ovário, tornando-se de extrema importância em análises de populações com desenvolvimento de neoplasias (INCA, 2019).

O cálculo de índice de massa corpórea (IMC) revelou que 36,7\% da amostra como pré-obesa e $28 \%$ como obesa de grau I. Como já descrito, a obesidade é um fator de risco para o desenvolvimento da doença analisada. O perfil de IMC observado está de acordo com os resultados de Morrison et al. (2018), que descreveram sua população como majoritariamente pré-obesa e obesa.

Considerando o histórico familiar de câncer, $63,7 \%$ da população apresentava casos familiares, sendo que 28,0\% das pacientes declararam histórico familiar de câncer de mama. Como descrito por Cardoso et al. (2019), a predisposição genética é considerada um dos principais fatores de risco para o desenvolvimento de câncer de mama.

A maioria das pacientes $(73,4 \%)$ declarava apresentar uma ou mais comorbidades, um total de 243 comorbidades foram relatadas, sendo as mais frequentes hipertensão (47,1\%), diabetes mellitus $(16,3 \%)$ e hipotireoidismo (3,8\%). Klastersky et al. (2000) apontam a presença de comorbidades severas como hipertensão e doença pulmonar obstrutiva crônica como fator de risco para o desenvolvimento de neutropenia febril.

O uso de medicamentos de forma contínua foi observado em 58,8\% da população. Foram utilizados 405 medicamentos, 16,8\% de ação nos grupos A (trato alimentar e metabolismo), 1,2\% no grupo B (sangue e órgãos formadores do sangue), 67,4\% no grupo C (sistema cardiovascular), 3,2\% no grupo H (preparações hormonais sistêmicas, excluindo hormônios sexuais e insulinas), $0,5 \%$ do grupo $\mathrm{M}$ (sistema musculoesquelético), 10,1\% do grupo $\mathrm{N}$ (sistema nervoso), $0,2 \%$ no grupo P (produtos antiparasitários, inseticidas e repelentes) e 0,5\% no grupo $\mathrm{R}$ (sistema respiratório). É notório o uso de medicamentos do grupo C, sendo 27,2\% de ação no sistema renina-angiotensina (C09) e 15,8\% de ação diurética (C03), de acordo com a classificação ATC (WHO, 2020), que representaram 43\% do total de medicamentos utilizados.

O risco de desenvolvimento de neutropenia está correlacionado a um conjunto de fatores, como o protocolo de quimioterapia utilizado, a idade do paciente, a presença de doença avançada, história prévia de neutropenia e persistência de eventos, ausência de profilaxia com antibióticos e/ou G-CSFs, presença de doença cardiovascular e performance status ruim (Klastersky et al., 2016; NCCN, 2020).

Observou-se que a população total ( $\mathrm{N}=289)$ fez uso de AC e docetaxel, sendo que na segunda parte do protocolo, 216 pacientes $(74,7 \%)$ fizeram uso de docetaxel sem PP e 73 pacientes $(25,3 \%)$ com PP.

De acordo com as diretrizes da National Comprehensive Cancer Network (NCCN) nos Estados Unidos, o esquema terapêutico T quando posterior ao protocolo AC em tratamento para câncer de mama é considerado de risco intermediário (10$20 \%$ ) para desenvolvimento de NF, sendo indicada a utilização de profilaxia primária com G-CSFs conforme alguns critérios relacionados ao paciente (NCCN, 2020). Não foi encontrada indicação de utilização de profilaxia com G-CSFs para neutropenia afebril conforme a classificação de risco do protocolo em estudo.

$\mathrm{Na}$ instituição onde o estudo foi realizado, a decisão de utilizar ou não a PP ficava a critério médico, não existindo um protocolo institucional para tal conduta.

A maioria das mulheres $(151-52,2 \%)$ apresentaram neutropenia durante a aplicação de AC, 137 sem presença de febre e 22 neutropenia febril, sendo que 8 pacientes apresentaram ambas, porém em aplicações distintas. Entre as pacientes que 
fizeram uso de docetaxel sem PP, 58 (26,9\%) apresentaram neutropenia durante a aplicação dessa etapa do protocolo, sendo 26 casos de neutropenia sem presença de febre e 32 casos de NF. Entre os pacientes que fizeram uso de docetaxel com PP, 11 pacientes $(15,1 \%)$ apresentaram neutropenia durante a aplicação de docetaxel, sendo 9 casos de neutropenia sem presença de febre e 2 casos de NF.

Kim et al. (2016) verificaram que 4,7\% das pacientes coreanas apresentaram NF durante o protocolo docetaxel (T), quando este era administrado sem PP com G-CSFs para tratamento de câncer de mama utilizando o esquema AC- T de quimioterapia. Destaca-se a escassez de estudos nacionais com o protocolo T no esquema AC-T, o que dificulta comparações desta ocorrência entre as pacientes brasileiras.

Das 289 participantes do estudo, $174(60,2 \%)$ apresentaram neutropenia ao longo do tratamento, sendo que 26,6\% apresentaram neutropenia apenas uma vez, 18,7\% apresentaram neutropenia duas vezes, 9,3\% apresentaram três vezes e 5,5\% apresentaram quatro ou mais vezes.

Foram realizados 1666 ciclos de quimioterapia, sendo 861 ciclos de AC e 805 ciclos de docetaxel. Durante a realização desses ciclos, foram observados 339 episódios de neutropenia, sendo 258 episódios durante os ciclos de AC e 81 episódios durante os ciclos de docetaxel - 59 episódios em pacientes que não utilizaram PP e 22 episódios em pacientes que utilizaram PP.

Os episódios de neutropenia foram classificados por gravidade e ciclos de quimioterapia. A Tabela 2 mostra os episódios durante os ciclos de AC.

Tabela 2: Episódios de neutropenia observados na aplicação de AC, segundo gravidade e ciclo (n=289).

\begin{tabular}{c|c|c|c|c|c}
\hline \multirow{2}{*}{ Grau de neutropenia } & \multicolumn{5}{|c}{ AC } \\
\cline { 2 - 6 } & Ciclo 1 & Ciclo 2 & Ciclo 3 & Ciclo 4 & Total $(\%)$ \\
\hline Grau 1 & 6 & 4 & 10 & 5 & $25(9,7)$ \\
\hline Grau 2 & 27 & 34 & 26 & 28 & $115(44,6)$ \\
\hline Grau 3 & 19 & 24 & 26 & 17 & $86(33,3)$ \\
\hline Grau 4 & 12 & 2 & 7 & 11 & $32(12,4)$ \\
\hline Total & 64 & 64 & 69 & 61 & 258 \\
\hline
\end{tabular}

Fonte: Autores.

A presença de eventos de neutropenia durante a administração do AC se mostra uniforme ao longo dos ciclos, com predomínio de graus 2 e 3.

A Tabela 3 mostra episódios de neutropenia durante os ciclos de docetaxel sem PP. 
Tabela 3: Episódios de neutropenia observados na aplicação de docetaxel, segundo gravidade e ciclo em pacientes que não utilizaram PP $(n=216)$.

\begin{tabular}{c|c|c|c|c|c}
\hline \multirow{2}{*}{ Grau de neutropenia } & \multicolumn{5}{|c}{ Docetaxel (T) } \\
\cline { 2 - 6 } & Ciclo 1 & Ciclo 2 & Ciclo 3 & Ciclo 4 & Total $(\%)$ \\
\hline Grau 1 & 0 & 0 & 1 & 0 & $1(1,7)$ \\
\hline Grau 2 & 4 & 0 & 2 & 2 & $8(13,6)$ \\
\hline Grau 3 & 2 & 3 & 3 & 1 & $9(15,3)$ \\
\hline Grau 4 & 25 & 9 & 4 & 3 & $41(69,5)$ \\
\hline Total & 31 & 12 & 10 & 6 & 59 \\
\hline
\end{tabular}

Fonte: Autores.

$\mathrm{Na}$ aplicação de docetaxel sem PP, diferente do esperado, a incidência de neutropenia não é cumulativa ao longo dos ciclos de quimioterapia, se mostrando maior no primeiro ciclo. Isto poderia ser explicado pela exposição inicial ao medicamento e pelo manejo da reação por parte dos oncologistas, o qual, dependendo da intervenção, pode influenciar na recorrência do episódio (Aarts et al., 2013; Pereira et al., 2015). Observamos predomínio de neutropenia grave (grau 4), a qual é limitante de dose e de grande preocupação, especialmente quando se trata doentes com alto risco de complicações mielotóxicas, como os pacientes em tratamento quimioterápico.

A Tabela 4 mostra episódios de neutropenia durante os ciclos de docetaxel com PP.

Tabela 4: Episódios de neutropenia observados na aplicação de docetaxel, segundo gravidade e ciclo em pacientes que utilizaram PP $(n=73)$.

\begin{tabular}{c|c|c|c|c|c}
\hline \multirow{2}{*}{ Grau de neutropenia } & \multicolumn{5}{|c}{ Docetaxel (T) } \\
\cline { 2 - 6 } & Ciclo 1 & Ciclo 2 & Ciclo 3 & Ciclo 4 & Total $(\%)$ \\
\hline Grau 1 & 2 & 1 & 1 & 0 & $4(18,2)$ \\
\hline Grau 2 & 6 & 3 & 4 & 2 & $15(68,2)$ \\
\hline Grau 3 & 1 & 1 & 0 & 1 & $3(13,6)$ \\
\hline Grau 4 & 0 & 0 & 0 & 0 & $0(0)$ \\
\hline Total & 9 & 5 & 5 & 3 & 22 \\
\hline
\end{tabular}

Fonte: Autores.

A presença de eventos de neutropenia durante a administração do docetaxel com PP se manteve predominantemente no primeiro ciclo, porém diferente dos pacientes que não utilizaram PP, nenhum evento de grau 4 foi observado, corroborando o efeito profilático dos G-CSFs frente a incidência de neutropenia severa.

A neutropenia é responsável por diminuir o êxito do tratamento quimioterápico, aumentando a necessidades de intervenções (Aras et al., 2020). Em uma revisão integrativa de Ferreira et al. (2017), foram descritas condutas como formas de tratamento de neutropenia febril em adultos. Nascimento, Andrade, Oliveira, Almeida and Gozzo, (2014), relataram suspensão, 
adiamento de ciclos e redução de dose como manejos de neutropenia em seu estudo. $\mathrm{O}$ uso de antimicrobianos por via oral ou sistêmica com internação associada também é descrito por Klastersky et al. (2016), como maneiras para controle de neutropenia febril.

Neste estudo as intervenções foram definidas como manejos desse evento. Observa-se que das 174 pacientes que apresentaram neutropenia, 129 pacientes necessitaram de uma conduta para controle desta.

Os manejos foram separados de acordo com grupos, sendo eles pacientes com neutropenia afebril e febril sob tratamento com AC, docetaxel sem uso PP e docetaxel com uso de PP. O manejo "uso de G-CSFs" não foi computado para o grupo de pacientes que fez uso de G-CSFs como PP, já que compreenderia todos os pacientes do grupo.

Os manejos encontram-se descritos na tabela 5.

Tabela 5: Manejos observados, segundo grupos AC, docetaxel (T) sem PP e docetaxel (T) com PP, com apresentação de episódios de neutropenia afebril e febril ( $\mathrm{n}=174)$.

\begin{tabular}{|c|c|c|c|c|c|c|c|}
\hline \multirow{3}{*}{ Manejo } & \multicolumn{2}{|c|}{$\mathrm{AC}$} & \multicolumn{2}{|c|}{ Docetaxel (T) sem PP } & \multicolumn{2}{|c|}{ Docetaxel (T) com PP } & \multirow{3}{*}{ Total $(\%)$} \\
\hline & \multicolumn{2}{|c|}{ Neutropenia } & \multicolumn{2}{|c|}{ Neutropenia } & \multicolumn{2}{|c|}{ Neutropenia } & \\
\hline & Afebril & Febril & Afebril & Febril & Afebril & Febril & \\
\hline Adiar o Ciclo & 44 & 6 & 2 & 6 & 0 & 1 & $59(20,3)$ \\
\hline Redução de dose & 2 & 0 & 10 & 17 & 6 & 1 & $36(12,4)$ \\
\hline Uso de antibioticoterapia & 4 & 23 & 13 & 32 & 0 & 2 & $74(25,4)$ \\
\hline Uso de G-CSFs & 18 & 14 & 9 & 26 & - & - & $67(23,0)$ \\
\hline $\begin{array}{c}\text { Internação para controle de } \\
\text { neutropenia }\end{array}$ & 1 & 13 & 8 & 25 & 0 & 0 & $47(16,2)$ \\
\hline Suspensão do tratamento & 0 & 0 & 5 & 3 & 0 & 0 & $8(2,7)$ \\
\hline Total & 69 & 56 & 47 & 109 & 6 & 4 & 291 \\
\hline
\end{tabular}

Fonte: Autores.

Foram analisados 291 manejos realizados. Observou-se que o adiamento de ciclo é bastante utilizado como manejo na presença de neutropenia afebril sob tratamento com AC, correspondendo a 74,6\% das vezes em que esse manejo foi realizado.

A redução de dose se apresenta como uma ação marcante de manejo de neutropenia de pacientes em tratamento com docetaxel sem uso PP, correspondendo a $75 \%$ das realizações desse manejo, com 10 realizações $(27,8 \%)$ para manejo de neutropenia afebril e 17 realizações $(47,2 \%)$ como manejo de neutropenia febril. A preservação de intensidade de dose de quimioterapia é fundamental no tratamento do câncer de mama, sendo relacionada a maiores taxas de sobrevida livre de doença e sobrevida global (Ladwa et al., 2018).

$\mathrm{O}$ uso de antibioticoterapia como manejo se encontra pronunciado para o tratamento de neutropenia febril em pacientes sob uso de AC (31,1\% das utilizações desse manejo), e para o tratamento de neutropenia afebril e febril em pacientes sem uso PP que realizaram tratamento com docetaxel, compreendendo 17,6\% e 43,2\% das utilizações de antibióticos. 
Quanto ao uso de G-CSFs, 67 mulheres fizeram uso como tratamento de eventos de neutropenia, sendo que 26 delas utilizaram para controle de eventos de neutropenia febril durante a administração de docetaxel, o que pode ser justificado pela marcante incidência desse evento no grupo de pacientes que não utilizou PP, refletindo uma queda no número de pacientes com neutropenia ao longo dos ciclos como já visto na tabela 3.

A neutropenia febril foi responsável por 38 internações ao longo do período analisado (80,8\% das internações), sendo 13 delas para controle após aplicação de AC e 25 delas para controle após aplicação de docetaxel sem uso PP. Constata-se que $13,1 \%$ dos pacientes tratados foram submetidos à internação para controle de neutropenia febril, assim como o previamente estabelecido por Kawatkar et al. (2017) que encontrou uma taxa de internação entre 7 e $22 \%$.

A suspensão de tratamento durante a aplicação de docetaxel foi observada em 37 pacientes, sendo que somente 8 pacientes tiveram tratamento suspenso devido a episódios de neutropenia durante a aplicação de docetaxel, exclusivamente pacientes do grupo sem uso PP, mostrando o importante efeito protetor de suporte durante a administração de quimioterapia mielossupressora que essa classe de medicamentos apresenta (Ferreira et al., 2017; Furuya, 2019).

\section{Conclusão}

A ocorrência de eventos de neutropenia ao longo do tratamento quimioterápico para câncer de mama é iminente devido ao efeito mielotóxico inerente a esses medicamentos. Assim, a adoção de manejos para controle desse evento torna possível a execução do tratamento com o menor dano possível ao paciente. Todavia, a realização de alterações no regime de tratamento seja por adiamento, redução de dose, internações ou suspensão pode levar ao comprometimento do resultado final ou mesmo do tratamento.

Neste estudo, a ocorrência de neutropenia, aponta para a necessidade de definir protocolos de utilização de profilaxia primária e manejo da reação adversa. A implantação de protocolos propicia um controle mais eficiente da utilização de GCSFs, evitando-se atrasos entre os ciclos e eventuais reduções de doses, que podem comprometer a resposta ao tratamento, além de propiciar a resistência tumoral aos quimioterápicos.

Um futuro trabalho com usuários de AC-T se mostra interessante para que se possa avaliar melhor o perfil das pacientes que apresentaram neutropenia, e assim determinar os fatores de risco relacionados ao paciente para essa reação adversa, estipulando assim um protocolo para utilização de profilaxia primária com G-CSFs e manejo da neutropenia.

Algumas limitações do estudo se apresentaram principalmente devido à sua natureza retrospectiva, levando à impossibilidade de acompanhamento próximo de pacientes envolvidos na reação adversa estudada. Condutas como adiamento de ciclos, reduções de dose, utilização de G-CSFs e antibioticos e suspensão do tratamento foram tomadas por critério médico, impedindo a obtenção de medidas uniformes de tratamento.

\section{Referências}

Aarts, M. J., Peters, F. P., Mandigers, C. M., Dercksen, M. W., Stouthard, J. M., Nortier, H. J., ... Tjan-Heijnen, V. C. (2013). Primary granulocyte colonystimulating factor prophylaxis during the first two cycles only or throughout all chemotherapy cycles in patients with breast cancer at risk for febrile neutropenia. Journal of clinical oncology, 31(34), 4290-4296. doi.org/10.1200/JCO.2012.44.6229

Abotaleb, M., Kubatka, P., Caprnda, M., Varghese, E., Zolakova, B., Zubor, P., ... Büsselberg, D. (2018). Chemotherapeutic agents for the treatment of metastatic breast cancer: an update. Biomedicine \& pharmacotherapy, 101, 458-477. doi.org/10.1016/j.biopha.2018.02.108

Aras, E., Bayraktar-Ekincioglu, A., \& Kilickap, S. (2020). Risk assessment of febrile neutropenia and evaluation of g-csf use in patients with cancer: a real-life study. Supportive care in cancer, 28(2), 691-699. doi.org/10.1007/s00520-019-04879-x

Associação Brasileira para o Estudo da Obesidade e da Síndrome Metabólica. (2016). Diretrizes Brasileiras de Obesidade (4a ed.). https://abeso.org.br/wpcontent/uploads/2019/12/Diretrizes-Download-Diretrizes-Brasileiras-de-Obesidade-2016.pdf

Bines, J., Earl, H., Buzaid, A. C., \& Saad, E. D. (2014). Anthracyclines and taxanes in the neo/adjuvant treatment of breast cancer: does the sequence matter?. Annals of oncology, 25(6), 1079-1085. doi.org/10.1093/annonc/mdu007 
Blum, J. L., Flynn, P. J., Yothers, G., Asmar, L., Geyer, C. E., Jacobs, S. A., ... Wolmark, N. (2017). Anthracyclines in early breast cancer: the abc trials-usor 06-090, nsabp b-46-i/usor 07132, and nsabp b-49 (nrg oncology). Journal of clinical oncology, 35(23), 2647-2655. doi.org/10.1200/JCO.2016.71.4147

Bray, F., Ferlay, J., Soerjomataram, I., Siegel, R. L., Torre, L. A., \& Jemal, A. (2018). Global cancer statistics 2018: GLOBOCAN estimates of incidence and mortality worldwide for 36 cancers in 185 countries. CA: a cancer journal for clinicians, 68(6), 394-424. doi.org/10.3322/caac.21492

Cardoso, F., Kyriakides, S., Ohno, S., Penault-Llorca, F., Poortmans, P., Rubio, I. T., ... ESMO Guidelines Committee. (2019). Early breast cancer: ESMO clinical practice guidelines for diagnosis, treatment and follow-up. Annals of oncology, 30(8), 1194-1220. doi.org/10.1093/annonc/mdz173

Catala, G., Mebis, J., Jerusalem, G., Verhoeven, D., Awada, A., Bols, A., ... Machiels, J. P. (2020). Neutropenia management in patients receiving myelosuppressive polychemotherapy for early breast cancer in Belgium: brons study results. Acta clinica Belgica, 75(2), 128-135. doi.org/10.1080/17843286.2018.1563735

Faqeer, N. A., Mashni, O., Dawoud, R., Rumman, A., Hanoun, E., \& Nazer, L. (2017). Comparing the incidence of febrile neutropenia resulting in hospital admission between the branded docetaxel and the generic formulations. Journal of clinical pharmacology, 57(2), 275-279. doi.org/10.1002/jcph.803

Ferreira, J. N., Correia, L. R. B. R., Oliveira, R. M. d., Watanabe, S. N., Possari, J. F., \& Lima, A. F. C. (2017). Managing febrile neutropenia in adult cancer patients: an integrative review of the literature. Revista Brasileira de Enfermagem, 70(6), 1301-1308. doi.org/10.1590/0034-7167-2016-0247

Furuya Y. (2019). Early neutropenia on day 8 treated with adjuvant docetaxel-based chemotherapy in early breast cancer patients: putative mechanisms within the neutrophil pool system. PloS one, 14(4), e0215576. doi.org/10.1371/journal.pone.0215576

Hansson, E. K., \& Friberg, L. E. (2012). The shape of the myelosuppression time profile is related to the probability of developing neutropenic fever in patients with docetaxel-induced grade IV neutropenia. Cancer chemotherapy and pharmacology, 69(4), 881-890. doi.org/10.1007/s00280-011-1769-7

Hegg, R., Mattar, A., Matos-Neto, J. N., Pedrini, J. L., Aleixo, S. B., Rocha, R. O., ... van-Eyll-Rocha, S. (2016). A phase III, randomized, non-inferiority study comparing the efficacy and safety of biosimilar filgrastim versus originator filgrastim for chemotherapy-induced neutropenia in breast cancer patients. Clinics, 71(10), 586-592. doi.org/10.6061/clinics/2016(10)06

Ho, M. Y., \& Mackey, J. R. (2014). Presentation and management of docetaxel-related adverse effects in patients with breast cancer. Cancer management and research, 6, 253-259. doi.org/10.2147/CMAR.S40601

Instituto Nacional de Câncer José Alencar Gomes da Silva. (2019). Estimativa 2020: incidência de câncer no Brasil. Rio de Janeiro: INCA.

Kawatkar, A. A., Farias, A. J., Chao, C., Chen, W., Barron, R., Vogl, F. D., \& Chandler, D. B. (2017). Hospitalizations, outcomes, and management costs of febrile neutropenia in patients from a managed care population. Supportive care in cancer, 25(9), 2787-2795. doi.org/10.1007/s00520-017-3692-X

Kim, C. G., Sohn, J., Chon, H., Kim, J. H., Heo, S. J., Cho, H., ... Kim, G. M. (2016). Incidence of febrile neutropenia in korean female breast cancer patients receiving preoperative or postoperative doxorubicin/cyclophosphamide followed by docetaxel chemotherapy. Journal of breast cancer, 19(1), 76-82. doi.org/10.4048/jbc.2016.19.1.76

Klastersky, J., de Naurois, J., Rolston, K., Rapoport, B., Maschmeyer, G., Aapro, M., ... ESMO Guidelines Committee (2016). Management of febrile neutropaenia: ESMO Clinical Practice Guidelines. Annals of oncology, 27(suppl 5), v111-v118. doi.org/10.1093/annonc/mdw325

Klastersky, J., Paesmans, M., Rubenstein, E. B., Boyer, M., Elting, L., Feld, R., ... Talcott, J. (2000). The Multinational Association for Supportive Care in Cancer risk index: A multinational scoring system for identifying low-risk febrile neutropenic cancer patients. Journal of clinical oncology, 18(16), 30383051. doi.org/10.1200/JCO.2000.18.16.3038

Ladwa, R., Kalas, T., Pathmanathan, S., Woodward, N., Wyld, D., \& Sanmugarajah, J. (2018). Maintaining dose intensity of adju vant chemotherapy in older patients with breast cancer. Clinical breast cancer, 18(5), e1181-e1187. doi.org/10.1016/j.clbc.2018.04.016

Minckwitz, G. von, Schwenkglenks, M., Skacel, T., Lyman, G. H., Pousa, A. L., Bacon, P., ... Aapro, M. S. (2009). Febrile neutropenia and related complications in breast cancer patients receiving pegfilgrastim primary prophylaxis versus current practice neutropaenia management: results from an integrated analysis. European journal of cancer, 45(4), 608-617. doi.org/10.1016/j.ejca.2008.11.021

Morrison, V. A., McCall, L., Muss, H. B., Jatoi, A., Cohen, H. J., Cirrincione, C. T., ... Hurria, A. (2018). The impact of actual body weight-based chemotherapy dosing and body size on adverse events and outcome in older patients with breast cancer: results from cancer and leukemia group b (calgb) trial 49907 (alliance a151436). Journal of geriatric oncology, 9(3), 228-234. doi.org/10.1016/j.jgo.2017.11.007

Nascimento, T. G. do, Andrade, M. de, Oliveira, R. A. de, Almeida, A. M. de, \& Gozzo, T. de O. (2014). Neutropenia: occurrence and management in women with breast cancer receiving chemotherapy. Revista Latino-Americana de Enfermagem, 22(2), 301-308. doi.org/10.1590/0104-1169.3305.2416

National Comprehensive Cancer Network. (2020). $\quad$ Myeloid $\quad$ Growth http://www.nccn.org/professionals/physician_gls/pdf/growthfactors.pdf.

Pereira, A. S., Shitsuka, D. M., Pereira, F. J., \& Shitsuka, R. (2018). Metodologia da pesquisa científica. Santa Maria, RS: UAB/NTE/UFSM. https://www.ufsm.br/app/uploads/sites/358/2019/02/Metodologia-da-Pesquisa-Cientifica_final.pdf

Pereira, L. C., Nogueira, T. A., Barbosa, L. A., Calil-Elias, S., Castilho, S. R. de. (2015). Adverse reactions to docetaxel: an active survey. Brazilian Journal of Pharmaceutical Sciences, 51, 551-559.doi.org/10.1590/S1984-82502015000300007.

Sociedade Brasileirade Farmacêuticos em Oncologia. (2011). Guia para notificação de reações adversas em oncologia (2a ed.). São Paulo: Conectfarma Publicações Científicas. 
Research, Society and Development, v. 10, n. 6, e48210616018, 2021

(CC BY 4.0) | ISSN 2525-3409 | DOI: http://dx.doi.org/10.33448/rsd-v10i6.16018

Wang, L., Baser, O., Kutikova, L., Page, J. H., \& Barron, R. (2015). The impact of primary prophylaxis with granulocyte colony-stimulating factors on febrile neutropenia during chemotherapy: a systematic review and meta-analysis of randomized controlled trials. Supportive care in cancer, 23(11), 3131-3140. doi.org/10.1007/s00520-015-2686-9

World Health Organization. (2020). The ATC classification structure and principles. http://www.whocc.no/atc_ddd_index/. 